\title{
Performance Evaluation of Beacon Enabled IEEE 802.15.4 MAC for Mobile Wireless Sensor Networks under NS-2
}

\author{
Muhammad Javed, Kartinah Zen and Halikul Bin Lenando \\ Faculty of Computer Sciences and Information Technologies, \\ University Malaysia Sarawak Kuching, Malaysia \\ javed.sikandry@gmail.com, \{kartinah and cool\}@fit.unimas.my
}

\begin{abstract}
Wireless Sensor Network (WSN) has a large number of nodes capable of sensing, communicating and computing. WSNs have limitations due to limited storage, processing and transmission power. The IEEE802.15.4 Medium Access Control (MAC) protocol is used for low-rate wireless personal area network (LR-WPAN). LR-WPAN is basically designed for static wireless sensor networks. However, from literatures, we observed that IEEE802.15.4 is able to support weak mobility in mobile sensor networks [7]. This paper evaluates the IEEE802.15.4 MAC for strong mobility in mobile sensor network environments. We evaluate the performance of IEEE802.15.4 MAC based on both static and mobile coordinators, and taking into account two parameters which are speed and number of beacon orders. We observed the effect on association period, disassociation, and synchronization between the mobile node and the coordinator in strong mobility of mobile nodes. From the experiments, we obtained results on throughput, association and synchronization with different speed and beacon orders. We found that the IEEE802.15.4 cannot maintain association period in strong mobility. The weaknesses of mobile node association attempt and synchronization process degrade the overall performance of a network. We also identify some research problems that need to be addressed for successful implementation of MAC protocol with strong mobility in Mobile Wireless Sensor Networks.
\end{abstract}

Keywords - wireless sensor network; IEEE802.15.4; Medium Access Control; performance evaluation

\section{INTRODUCTION}

Wireless Sensor Network (WSN) is a group of locally dispersed and dedicated sensors for monitoring and recording the physical conditions of the environment and organizing the collecting data at the central location [1]. The basic idea of IEEE802.15.4 is to support short range applications like habitat monitoring, battlefield surveillance, intelligent transportation system, nuclear, biological, and chemical attack detection, industry and home automation and many more[1]. Such applications require a small, low-cost, highly reliable technology that offer a long life battery, IEEE and ZigBee Alliance [2] are working together to achieve this objective. IEEE802.15.4 standard focuses on the specification of the lower layers (Physical and Medium Access Control Layer), whereas ZigBee alliance provides upper layers of the protocol stack. [3].

WSN is mainly designed for static sensor networks. Introducing mobility concept in WSN raises some serious

\author{
Hushairi Zen \\ Faculty of Engineering, \\ University Malaysia Sarawak Kuching, Malaysia \\ zhushair@feng.unimas.my
}

research problems. Solution to these research problems is a challenge for research community. Node mobility will enhance the application capability of low-rate wireless personal area networks (LR-WPAN). Coordinator and node mobility will facilitate different applications from industry and home automation to target detection, floods, earthquake, battlefield surveillance, intelligent transportation system and animal monitoring. This paper encounters the issues that how coordinator and sensor node mobility affects network performance at Medium Access Control (MAC) layer. This is important to provide smooth flow of traffic without affecting the LR-WPAN features. The task of MAC protocol is to transmit data efficiently among nodes [4]. The standard IEEE802.15.4 MAC protocol has been evaluated for low data-rate and low power-consumption networks in the static environment and the results proven the suitability of IEEE802.15.4 for LR-WPAN [5][6]. In this paper, IEEE802.15.4 MAC protocol is evaluated for mobile sensor networks. The results proven that node mobility decreases the performance of nodes in IEEE802.15.4.

This paper is organized as follows: in the next section, we provide the literature review. In section III, the IEEE802.15.4 is briefly explained. In this section the association and synchronization process of IEEE802.15.4 is also explained. In section IV we explain the mobile wireless sensor networks scenarios for experiments. First scenario has mobile node and static coordinators while second scenario has mobile node and mobile coordinators. Section $\mathrm{V}$ has simulation results and last section VI concludes the paper.

\section{LITERATURE REVIEW}

Introducing mobility features in both coordinators and nodes in WSNs is a complicated work. There are number of papers[7] [8] in literature that focus on node mobility at MAC layer in WSNs and few papers focus on mobility in IEEE802.15.4 MAC protocol for mobile sensor networks [7]. MS-MAC [9] is a mobility-aware MAC protocol for sensor networks. MS-MAC is the extension of S-MAC to support mobility. MS-MAC does not consider the problem of reliable communication of mobile nodes and network coverage. S-MAC [10], Sensor MAC has introduced lowduty cycle operation in multi-hop WSNs. In S-MAC, nodes spend most time in sleep mode to reduce energy consumption but it does not consider node mobility. MMAC [11] is mobility adaptive, collision free MAC protocol. M-MAC is a schedule-based MAC protocol. MMAC includes a flexible frame time that enables the protocol to dynamically adopt the weak mobility. 